BULL. AUSTRAL. MATH. SOC.

VOL. 25 (1982), 1-28.

\title{
ON ORBITS OF ALGEBRAIC GROUPS \\ AND LIE GROUPS
}

\author{
R.W. RICHARDSON
}

\begin{abstract}
In this paper we will be concerned with orbits of a closed subgroup 2 of an algebraic group (respectively Lie group) $G$ on a homogeneous space $X$ for $G$. More precisely, let $D$ be a closed subgroup of $G$ and let $X$ denote the coset space $G / D$. Let $S$ be a subgroup of $G$ and let $Z$ denote $\left(G^{S}\right)^{0}$, the identity component of $G^{S}$, the centralizer of $S$ in $G$. We consider the orbits of $Z$ on $X^{S}$, the set of fixed points of $S$ on $X$. We also treat the more general situation in which $S$ is an algebraic group (respectively Lie group) which acts on $G$ by automorphisms and acts on $X$ compatibly with the action of $G$; again we consider the orbits of $\left(G^{S}\right)^{0}$ on $X^{S}$.
\end{abstract}

Our main results are the following theorems:

THEOREM A. Let $G$ be a closed normal connected subgroup of the affine algebraic group $H$. Let $H$ act morphically on an algebraic variety $X$ and assume that the induced action of $G$ on $X$ is transitive. Let $S$ be a closed subgroup of $B$ and let $Z=\left(G^{S}\right)^{0}$. Assume that the following two conditions are satisfied:

(i) the adjoint representation of $S$ on $\underline{\underline{g}}$, the Lie algebra of $G$, is semisimple; and

Received 20 July 1981. 
(ii) the Lie algebra of 2 is $\underline{\underline{8}}^{S}$.

Then $Z$ acts transitively on each connected component of $x^{S}$. In particular, $Z$ has only a finite number of orbits on $x^{S}$ and each such Z-orbit is closed.

THEOREM B. Let $G$ be a closed connected normal subgroup of the lie group $H$. Let $H$ act differentiably on a differentiable manifold $X$ such that the induced action of $G$ on $X$ is transitive. Let $S$ be $a$ subgroup of $H$ such that the adjoint representation of $S$ on $\underline{\underline{g}}$ is semisimple. Then $X^{S}$ is a closed differentiable submanifold of $X$ and $Z=\left(G^{S}\right)^{0}$ acts transitively on each connected component of $x^{S}$.

THEOREM C. Let $G$ be a closed connected normal subgroup of the affine algebraic group $H$ and assume that $G$ is reductive. Let $S$ be a inearly reductive, closed subgroup of $H$ and let $z=\left(G^{S}\right)^{0}$. Let $H$ act morphically on the affine algebraic variety $X$ and let $x \in X^{S}$. Let $C$ denote the unique closed G-orbit in the closure of the orbit $G \cdot x$. Then there exists a (multiplicative) one-parameter subgroup $\lambda$ of $z$ such that $\lim \lambda(t) \cdot x$ exists and is a point of $C$. In particular, $G \cdot x$ is $t \rightarrow 0$ closed if and only if $z \cdot x$ is closed.

The formulation and proof of Theorem A were worked out in several conversations with $\mathrm{V}$. Kăc and this theorem should be regarded as joint work. The proof uses an idea from [13]. This theorem seems to be quite useful in a number of rather technical problems involving orbits of algebraic groups. We remark that conditions (i) and (ii) of Theorem A hold whenever $S$ is a linearly reductive group (see Section 4 ). In Theorem A the Z-orbits on $X^{S}$ do not necessarily all have the same dimension, so that the geometric picture is more complicated than it might appear at first glance.

Theorem B is a Lie group version of Theorem A. The idea of the proof is the same, although the technical details are a bit more complicated.

The proof of Theorem $C$ depends on a strengthened form, due to Kemp $f$ [8], of the Hilbert-Mumford Theorem. If the base field is of 
characteristic zero, then Theorem $C$ follows from a result of Luna [9], so that Theorem $\mathrm{C}$ is new only for the case of prime characteristic.

Several applications of our theorems to involutions of algebraic groups are given in Section 9. These results will be used in an essential way in a forthcoming paper by the author on involutions of reductive algebraic groups. A few other applications are discussed in section 10.

\section{Preliminaries}

Our basic reference for algebraic groups is the book by Borel [1]. In general, we will follow the notation and terminology therein. All algebraic groups and algebraic varieties are taken over an algebraically closed field $k$. We will usually denote the lie algebra of an algebraic group (respectively Lie group) $G, H, K$, and so on, by the corresponding lower case letter with a double underline $\underline{\underline{g}}, \underline{\underline{h}}, \underline{\underline{k}}$, and so on. All algebraic groups considered will be affine algebraic groups.

Let $G$ be a group and let $X$ be a $G$-set. If $g \in G$ and $x \in X$, then $g \cdot x$ denotes the image of $x$ under the action of $g, G \cdot x$ denotes the G-orbit of $x$ and $G_{x}$ denotes the isotropy subgroup of $G$ at $x$.

If $S$ is a subset of $G$, we write $X^{S}$ for the set of fixed points of $S$ on $X: X^{S}=\{x \in X \mid g \cdot x=x$ for every $g \in S\}$. For $s \in G$, we write $X^{s}$ instead of $X^{\{s\}}$. We frequently consider $G$ as a $G$-set, where $G$ acts by inner automorphisms. Thus if $S$ is a subset of $G, G$ denotes the centralizer of $S$ in $G$. If $L$ is a subset of $G$ and $g \in G$, we often write $g_{L}$ for $g L g^{-1}$.

If the algebraic group $G$ acts morphically on the algebraic variety $X$, we say that $X$ is a G-variety. If the algebraic group $S$ acts morphically on the algebraic group $G$ and if, for every $s \in S$, the morphism $x \mapsto s \cdot x$ is an automorphism of $G$, then we say that $G$ is an $S$-group. Let $G$ be an algebraic group, let $X$ be a $G$-variety, let $a \in X$ and let $C$ denote the orbit $G \cdot a$. For $x \in C$, let $\varphi_{x}: G+C$ denote the orbit map: $\varphi_{x}(g)=g \cdot x$. We say that $G$ acts separably on the orbit $C$ if $\varphi_{a}$ is a separable morphism; if $\varphi_{a}$ is separable, then $\varphi_{x}$ 
is separable for every $x \in C$.

\section{Actions of semi-direct products}

In this paper we consider the action of a closed subgroup $Z$ of an algebraic group (respectively Lie group) $G$ on a coset space $G / D=X$. However, we can extend the scope of our results by considering an algebraic group (respectively Lie group) $H$ which contains $G$ as a closed normal subgroup and is such that the action of $G$ on $X$ extends to an action of $H$. This is essentially equivalent to considering the action of a semidirect product $G \times S$ on $X$, where $G$ is an $S$-group. In this section, we discuss several elementary constructions involving actions of semidirect products which will be used in the sequel. We confine our discussion to algebraic groups. However, all of our constructions carry over to the case of Lie groups with no essential changes.

Let $S$ and $G$ be algebraic groups and let $G$ be an $S$-group. We denote by $G \ltimes S$ the semi-direct product of $G$ and $S$; the underlying algebraic variety of $G \propto S$ is $G \times S$ and multiplication is defined by $\left(g_{1}, s_{1}\right)\left(g_{2}, s_{2}\right)=\left(g_{1}\left(s_{1} \cdot g_{2}\right), s_{1} s_{2}\right)$. We consider $G$ and $S$ as closed subgroups of $G \propto S$ in the obvious way. Assume that $G$ and $S$ act morphically on an algebraic variety $X$. We say that the actions of $G$ and $S$ on $X$ are compatible if

$$
s \cdot(g \cdot x)=(s \cdot g) \cdot(s \cdot x) \text { for } s \in S, g \in G \text { and } x \in X \text {. }
$$

Let $G$ and $S$ act compatibly on $X$. We define a morphism

$$
\eta:(G \propto S) \times X \rightarrow X
$$

by $n((g, s), x)=g \cdot(s \cdot x)$. Set $\eta((g, s), x)=(g, s) \cdot x$. Then an easy computation using (2.1) shows that

$$
\left(g_{1}, s_{1}\right) \cdot\left(\left(g_{2}, s_{2}\right) \cdot x\right)=\left(g_{1}\left(s_{1} \cdot g_{2}\right), s_{1} s_{2}\right) \cdot x \text {. }
$$

Hence $\eta$ defines a morphic action of $G \times S$ on $X$. This is the unique action of $G \times S$ on $X$ which extends the actions of $G$ and $S$. Conversely, the restrictions to $G$ and $S$ of an action of $G \propto S$ on $X$ are compatible.

Now let $G$ be an $S$-group and let $D$ be an $S$-stable closed subgroup of $G$. If $s \in S$ and $g \in G$, we set $s \cdot(g D)=(s \cdot g) D$; equivalently 
$s \cdot(g D)$ is the $s$-image of the left coset $g D$ under the action of $S$ on $G$. Clearly this defines an action of $S$ on (the set) $G / D$. A standard argument using the properties of quotient morphisms (see [1, pp. 172-186]) shows that the map

$$
S \times(G / D) \rightarrow G / D, \quad(s, g D) \mapsto s \cdot(g D),
$$

is a morphism of algebraic varieties. Hence we have defined a morphic action of $S$ on $G / D$. We let $G$ act on $G / D$ by left translations. One checks immediately that the actions of $G$ and $S$ on $G / D$ are compatible and hence determine a morphic action of the semi-direct product $G \propto S$ on $G / D$.

REMARK. One needs to be a bit careful in the above construction. Let $S$ be a closed subgroup of $G$ and let $S$ act on $G$ by inner automorphisms. This defines an $S$-group structure on $G$. Let $D$ be a closed subgroup of $G$ normalized by $S$. Then the action of $S$ on $G / D$ by left translations is not the same as the above defined action of $S$ on $G / D$ unless $S$ is contained in $D$.

\section{Proof of Theorem A}

We will use the following lemma, which is an abstraction of a method used in [13] (see also [18, p. 469]):

LEMMA 3.1. Let the algebraic group $G$ act morphically on the algebraic variety $X$. Let $Z$ be a closed connected subgroup of $G$ and let $Y$ be a closed Z-stable subvariety of $X$. Assume that, for every $y \in Y, T_{y}(G \cdot y) \cap T_{y}(y) \subset\left(d \varphi_{y}\right)_{e}(\underline{\underline{z}})$, where $\varphi_{y}: G \rightarrow G \cdot y$ is the orbit map. Then, for every $y \in Y,(G \cdot y) \cap Y$ is a finite union of 2-orbits and $Z$ acts transitively on each irreducible component of $(G \cdot y) \cap Y$. Moreover, for $y \in Y, Z$ 'acts separably on the orbit $Z \cdot y$.

Proof. Let $y \in Y$ and let $C$ be an irreducible component of $(G \cdot y) \cap Y$ which contains $y$. Clearly $z \cdot y$ is contained in $C$. We have

$$
T_{y}(C) \subset T_{y}(G \cdot y) \cap T_{y}(Y) \subset\left(d \varphi_{y}\right) e(\underline{\underline{z}}) \subset T_{y}(Z \cdot y) \subset T_{y}(C)
$$

and hence $T_{y}(C)=T_{y}(z \cdot y)$. Since $z \cdot y$ is a smooth variety, it follows that 


$$
\operatorname{dim} C \leq \operatorname{dim} T_{y}(C)=\operatorname{dim} T_{y}(z \cdot y)=\operatorname{dim} z \cdot y
$$

Consequently $z \cdot y$ is an open neighbourhood of $y$ in $C$. It follows immediately that $Z$ acts transitively on $C$. It is also clear that $Z$ acts separably on the orbit $2 \cdot y$.

Proof of Theorem A. Let the notation be as in the statement of Theorem A. First we consider the case in which $G$ acts separably on the orbit $X$. Let $Y=X^{S}$. Since $Z$ centralizes $S, Y$ is 2-stable. Let $y \in Y$ and let $D=G_{y}$. Then the orbit map $\varphi_{y}: G \rightarrow G \cdot y=X$ induces a $G$-isomorphism of the coset space $G / D$ onto $X$. Let $\psi$ denote the differential $\left(d \varphi_{y}\right)_{e}: \underline{\underline{g}} \rightarrow T_{y}(X)$. Then $\psi$ is surjective. Since $S \subset H_{y}, S$ acts on the tangent space $T_{y}(X)$. If $s \in S$ and $g \in G$, then

$$
\varphi_{y}\left(s g s^{-1}\right)=\left(s g s^{-1}\right) \cdot y=s \cdot(g \cdot y)=s^{\bullet} \varphi_{y}(g) \text {. }
$$

Hence $\varphi_{y}$ is an $S$-morphism. It follows easily that $\psi: \underline{\underline{g}} \rightarrow T_{y}(X)$ is an $S$-module homomorphism (here $S$ acts on $\underline{\underline{g}}$ via the adjoint representation). Consequently $\underline{\underline{\mathrm{d}}}=\operatorname{kernel}(\psi)$ is an $S$-submodule of $\underline{\underline{g}}$. Since $\underline{\underline{g}}$ is a semisimple $S$-module, there exists an $S$-submodule $\underline{\underline{q}}$ of $\underline{\underline{g}}$ such that $\underline{\underline{\mathrm{g}}}=\underline{\underline{\mathrm{a}}} \oplus \underline{\underline{\mathrm{q}}} \cdot$ We clearly have $\underline{\underline{\mathrm{z}}}=\underline{\underline{\mathrm{g}}}^{S}=\underline{\underline{\mathrm{a}}} \Phi \underline{\underline{\mathrm{q}}}{ }^{S}$. Hence we see that $\psi$ determines an $S$-module isomorphism of $\underline{\underline{q}}$ onto $T_{y}(X)$ and that $\psi(\underline{\underline{z}})=\psi\left(\underline{\underline{q}} \underline{\underline{q}}^{S}=T_{y}(X)^{S}\right.$. Clearly $\psi(\underline{\underline{z}}) \subset T_{y}(Z \cdot y)$. But since $Y=X^{S}$, we have $T_{y}(Y) \subset T_{y}(X)^{S}$. Thus we have $T_{y}(G \cdot y) \cap T_{y}(Y) \subset T_{y}(X)^{S}=\psi(\underline{\underline{z}})$. The conclusion of Theorem A now follows from Lemma 3.1.

Now for the case where the action of $G$ on $X$ is not necessarily separable. We may assume that $x^{S}$ is non-empty. Let $y \in x^{S}$ and let $D=G_{y}$. Then $S$ normalizes $D$. Since $S$ normalizes $G$, we may consider $G$ as an $S$-group with $S$ acting on $G$ by conjugation. As in Section 2, we define a morphic action of the semi-direct product $G \propto S=K$ on $G / D$. Since $G$ acts separably on $G / D$, it follows from the case of Theorem A just proved (with $H$ replaced by $K$ ) that $Z$ acts transitively 
on each irreducible component of $(G / D)^{S}$. The orbit map $\varphi_{y}: G \rightarrow G \cdot y=X$ determines a bijective $G$-morphism $\alpha: G / D \rightarrow X$. Since $G / D$ and $X$ are normal varieties, it follows from Zariski's Main Theorem (see [5, p. 138, Corollary 3]) that $\alpha$ is a finite morphism. In particular $\alpha$ is a closed map. Consequently $\alpha$ is a homeomorphism. We have

$$
\alpha(s \cdot(g D))=\alpha\left(s g s^{-1} D\right)=\left(s g s^{-1}\right) \cdot y=s \cdot(g \cdot y)=s \cdot \alpha(g D) .
$$

Hence $\alpha$ is an $S$-morphism. Since $Z$ acts transitively on each irreducible component of $(G / D)^{S}$, we see immediately that $Z$ acts transitively on each irreducible component of $x^{S}$. This proves Theorem A.

We record for later use the following result, which was proved in the course of the above proof.

COROLLARY 3.2. Let the notations and assumptions be as in Theorem A and assume that $G$ acts separably on $X$. Then, for $y \in X^{S}, Z$ acts separably on the orbit $2 \cdot y$.

3.3. An example. Let $G=H=\mathrm{GL}_{3}(k)$, let $X$ denote the projective plane $P_{2}(k)$ and let $G$ act on $X$ in the usual way. Let $S=\left\{\operatorname{diag}(t, t, 1) \mid t \in k^{*}\right\} ; S$ is a one-dimensional algebraic torus in $G$. Clearly $S$ satisfies conditions $(i)$ and (ii) of Theorem A. Now $X^{S}$ consists of two connected components, one a projective line and one a point. Consequently we see that in Theorem A the irreducible components of $x^{S}$ do not necessarily all have the same dimension.

\section{A lemma on linearly reductive groups}

We recall that an affine algebraic group $S$ is linearly reductive if every rational representation of $S$ is semisimple. In characteristic zero, $S$ is linearly reductive if and only if the identity component $S^{0}$ is a reductive algebraic group. In prime characteristic $p$, it has been shown by Nagata [11] that $S$ is linearly reductive if and only if $S^{0}$ is a torus and the order of $S / S^{0}$ is relatively prime to $p$.

Let $G, H$ and $S$ be as in the statement of Theorem A. We wish to 
show that conditions $(i)$ and (ii) of Theorem A hold if $S$ is linearly reductive. It follows immediately from the definition of linearly reductive groups that condition ( $i$ ) holds. Condition (ii) will follow from the following lemma:

LEMMA 4.1. Let $S$ and $G$ be algebraic groups with $S$ a linearly reductive group and $G$ an $S$-group. Let $z=\left(G^{S}\right)^{0}$. Then $\underline{\underline{\mathrm{z}}}=\underline{\underline{\underline{g}}}{ }^{S}$.

For the proof of Lemma 4.1, we will use the following lemma.

LEMMA 4.2. Let $E$ be a finite dimensional vector space over $k$ and

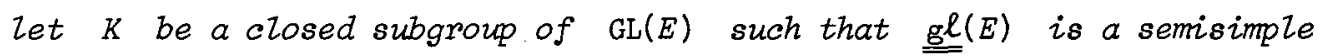
$K$-module. Let $M$ be a closed subgroup of $G L(E)$ which contains $K$. Then $\underline{\underline{m}}^{K}$ is the Lie algebra of $M^{K}$.

Proof. We will use Corollary 3.2 of Theorem A. We identify $\underline{\underline{g \ell}(E)}$ with $\operatorname{End}_{k}(E)$. Now $\left.\underline{\underline{g \ell(E)}}\right)^{K}$ is the centralizer of $K$ in $\operatorname{End}_{k}(E)$; in particular, $\underline{\underline{g \ell}}(E)^{K}$ is an associative subalgebra of $\operatorname{End}_{k}(E)$. Moreover $\mathrm{GL}(E)^{K}=C$ is simply the intersection of $\mathrm{GL}(E)$ with $\underline{\underline{\mathrm{g} \ell}(E)^{K}}$, hence $C$ is an open subset of $\underline{\underline{g} \ell(E)^{K}}$. Clearly $\underline{\underline{c}}=\underline{\underline{g} \ell(E)^{K}}$ is the Lie algebra of $C$. We can apply Corollary 3.2 to the action of $C$ on $(\mathrm{GL}(E) / M)^{K}$. Let $\pi: \mathrm{GL}(E) \rightarrow \mathrm{GL}(E) / M$ be the canonical map and let $y=\pi(e)$. Then $y \in(\mathrm{GL}(E) / M)^{K}$ and hence, by Corollary 3.2, the orbit map $\varphi_{y}: C \rightarrow C \cdot y$, $\varphi_{y}(c)=c \cdot y$, is separable. But the kernel of $\left(d \varphi_{y}\right)_{e}: \stackrel{\underline{c}}{\rightarrow} T_{y}(\mathrm{GL}(E) / M)$ is just $\underline{\underline{c}} \cap \underline{\underline{m}}=\underline{\underline{m}}^{K}$ and the isotropy subgroup $C_{y}$ is $C \cap M=M^{K}$. Since $\varphi_{y}$ is separable, $\operatorname{dim} M^{K}=\operatorname{dim} \underline{\underline{m}}^{K}$ and therefore $\underline{\underline{m}}^{K}$ is the Lie algebra of $M^{K}$.

Now for the proof of Lemma 4.1. We may embed the semi-direct product $G \propto S=M$ as a closed subgroup of some $G L(E)$. We consider $G$ and $S$ as closed subgroups of $M$, hence as closed subgroups of $\mathrm{GL}(E)$. Since $S$ is linearly reductive, $\underline{g \ell}(E)$ is a semisimple $S$-module. By Lemma 4.2, $\operatorname{dim} M^{S}=\operatorname{dim} \underline{\underline{m}}$. But it is trivial to show that $M^{S}=G^{S} \times S^{S}$ (as an algebraic variety) and that $\stackrel{\underline{\underline{m}}}{=}=\underline{\underline{g}} \times \underline{\underline{s}}$ (as a vector space). Since 
$\operatorname{dim} G^{S} \leq \operatorname{dim} \underline{\underline{\mathrm{g}}}$ and $\operatorname{dim} S^{S} \leq \operatorname{dim} \underline{\underline{\underline{s}}}$, we must have $\operatorname{dim} G^{S}=\operatorname{dim} \underline{\underline{\underline{g}}}{ }^{S}$. If $z=(G)^{0}$, it follows that $\underline{\underline{z}}=\underline{\underline{g}}$.

REMARK. The case of Lemma 4.1 in which $S$ is a diagonalizable group is treated in [16, p. 125, Corollary 4.4.7] by a different method.

\section{Proof of Theorem B}

For the proof of Theorem B, we will use the following lemma:

LEMMA 5.1. Let $U$ and $V$ be differentiable manifolds, let $n$ be a positive integer, let $f: U \rightarrow V$ be a differentiable map, and let $n_{j}: V \rightarrow \mathrm{R}^{n}$ be differentiable maps, $j=1, \ldots, m$. Let $a \in U$ and let $y=f(a)$. Assume that $\eta_{j}(f(U))=$ lo\} for $j=1, \ldots, m$. Let $M=\left\{v \in V \mid \eta_{j}(v)=0\right.$ for $\left.j=1, \ldots, m\right\}$ and let $E \subset T_{y}(V)$ denote the intersection of the kermels of the differentials $\left(d n_{j}\right)_{y}: T_{y}(V) \rightarrow \mathrm{R}^{n}$, $j=1, \ldots, m$. Assume that the differential $d f_{a}: T_{\alpha}(U) \rightarrow T_{y}(V)$ has image $E$. Then $f(U)$ is a neighbourhood of $y$ in $M$.

Lemma 5.1 is a straightforward application of the inverse function theorem. See [12, p. 91, Lemma 6.8] for a detailed proof in a slightly more general setting.

Now for the proof of Theorem B. We use the notation in the statement of the theorem. Let $n=\operatorname{dim} X$ and let $y \in X^{S}$. We wish first to show that the orbit $z \cdot y$ is an open neighbourhood of $y$ in $X^{S}$. Since $y \in X^{S}, S$ acts on $T_{y}(X)$. We choose a finite set $\left\{s_{1}, \ldots, s_{m}\right\}$ of elements of $S$ such that $T_{y}(X)^{S}=\bigcap_{j=1}^{m} T_{y}(X)^{s} j$. Choose an open neighbourhood $E$ of $y$ in $X$ and a diffeomorphism $h$ of $E$ onto an open subset of $\mathrm{R}^{n}$ such that $h(y)=0$. Let $V$ be an open neighbourhood of $y$ in $E$ such that $s_{j} \cdot V \subset E$ for $j=1, \ldots, m$. We define differentiable maps $\eta_{j}: V \rightarrow \mathrm{R}^{n}, j=1, \ldots, m$, by $\eta_{j}(x)=h(x)-h\left(s_{j} \cdot x\right)$. We note that $x^{S} \cap V \subset \eta_{j}^{-1}(0)$. We also note 
that the differential $\left(d n_{j}\right)_{y}: T_{y}(V) \rightarrow \mathrm{R}^{n}$ is given by $\left(d n_{j}\right)_{y}(v)=d h_{y}\left(v-s_{j} \cdot v\right)$. Since $d h_{y}$ is a linear isomorphism, kernel $\left(d n_{j}\right)_{y}=T_{y}(X)^{s} j$. Consequently we obtain

$$
\prod_{j=1}^{m} \operatorname{kernel}\left(d n_{j}\right)_{y}=T_{y}(X)^{S}
$$

Let $D$ denote the isotropy subgroup $G_{y}$. Then the orbit map $\varphi_{y}: G \rightarrow X$ induces a $G$-diffeomorphism of $G / D$ onto $X$. In particular $\left(d \varphi_{y}\right)_{e}: \underline{\underline{g}} \rightarrow T_{y}(X)$ is a surjection; let $\psi=\left(d \varphi_{y}\right)_{e}$. The argument given in Section 3 shows that $\psi$ is an $S$-module homomorphism and that $\psi(\underline{\underline{z}})=\psi\left(\underline{\underline{g}}{ }^{S}\right)=T_{y}(X)^{S}$. Let $U=\left\{z \in Z \mid \varphi_{y}(z) \in V\right\}$ and let $f: U \rightarrow V$ denote the restriction of $\varphi_{y}$ to $U$. The image of $d f_{e}$ is $\psi(\underline{\underline{z}})=T_{y}(X)^{S}=\bigcap_{j=1}^{m} \operatorname{kernel}\left(d \eta_{j}\right)_{y}$. It now follows from Lemma 5.1 that $f(U)$ is a neighbourhood of $y$ in $n n_{j}^{-1}(0)$. Since $f(U) \subset z \cdot y$, we see that $z \cdot y$ is an open neighbourhood of $y$ in $x^{S}$.

We have shown that, for every $y \in x^{S}, z \cdot y$ is a neighbourhood of $y$ in $X^{S}$. It follows that each $Z$-orbit on $X^{S}$ is both open and closed in $X^{S}$. Hence $Z$ acts transitively on each connected component of $X^{S}$. Since each 2-orbit on $x^{S}$ is closed in $x^{S}$, hence closed in $X$, it follows by a standard result (see [6, p. 62, 16.10.7]) that each such orbit is a closed differentiable submanifold of $X$. Since each such orbit is relatively open in $X^{S}$, we see that $X^{S}$ is a closed differentiable submanifold of $X$. This completes the proof of Theorem B.

REMARK. In Theorem B, one cannot necessarily conclude that $Z$ has only a finite number of orbits on $X^{S}$, that is that $X^{S}$ has only a finite number of connected components. However, in certain cases of Theorem $B$ involving real algebraic groups, one can show that $X^{S}$ has only a finite number of connected components. For example, let $H$ be a (complex) affine 
algebraic group defined over $\mathrm{R}$, let $\eta: H \rightarrow \mathrm{GL}(V)$ be a rational representation of $H$ defined over $\mathrm{R}$ and let $x$ be an R-rational point of $V$ (respectively $P(V)$ ). Let $K$ be a closed connected normal subgroup of $H$ defined over $\mathrm{R}$ and let $G$ denote the identity component of the real Lie group $K(\mathrm{R})$. Let $S$ be a subgroup of $H(\dot{R})_{x}$ such that the adjoint representation of $S$ on $\underline{\underline{g}}$ is semisimple and let $Z=G^{S}$. Let $X=G \cdot x$. Then $X$ is a locally closed differentiable submanifold of $V$ (respectively $P(V)$ ) and is $S$-stable. One can show that $X^{S}$ has only a finite number of connected components and hence that $Z$ has only a finite number of orbits on $X$. The main point of the proof is that, by a theorem of Whitney [20], if $Y$ is a Zariski locally-closed subset of $V(R)$ (respectively $P(V(R))$ ), then $Y$ has only a finite number of components (with respect to the topology on $Y$ determined by the usual topology of the real numbers). We omit further details.

\section{Another technical result on linearly reductive groups}

If the base field $k$ is of characteristic zero, then all of the results of this section are either well-known or follow readily from standard results. We shall assume throughout this section that $k$ is of prime characteristic $p$. If $S$ is a linearly reductive group, this will imply that $S^{0}$ is a torus and that $\left|S / S^{0}\right|$ is relatively prime to $p$.

The purpose of this section is to prove the following proposition:

PROPOSITION 6.1. Let $G$ be a closed connected normal subgroup of an algebraic group $H$ and let $S$ be a closed subgroup of $H$. Assume that $G$ is a reductive group and that $S$ is a linearly reductive group. Let $P$ be a parabolic subgroup of $G$ which is normalized by $S$. Then there exists a Levi subgroup $L$ of $P$ which is normalized by $S$.

6.2. Non-commutative cohomology of algebraic groups. The most convenient way to prove Proposition 6.1 seems to be via the non-commutative cohomology of algebraic groups. First we need to transpose some standard definitions and results from Galois cohomology to this slightly different framework. See [2] for Galois cohomology.

Let $G$ and $M$ be algebraic groups and assume that $M$ is a $G$-group. A morphism of algebraic varieties $\alpha: G \rightarrow M$ is a one-cocycle of $G$ with 
values in $M$ if $\alpha(g h)=\alpha(g)(g \cdot \alpha(h))$ for every $g, h \in G$. Let $Z^{l}(G, M)$ denote the set of all one-cocycles of $G$ with values in $M$. We define an action of $M$ on the set $Z^{\perp}(G, M)$ as follows: if $m \in M$ and $\alpha \in Z^{l}(G, M)$, then the one-cocycle $m \cdot \alpha$ is defined by $(m \cdot \alpha)(g)=m \alpha(g)(g \cdot m)^{-1}$ for $g \in G$. Two one-cocycles $\alpha$ and $\beta$ are cohomologous if there exists $m \in M$ such that $\beta=m \cdot \alpha$. The set of orbits of $M$ on $Z^{\perp}(G, M)$ is denoted by $H^{\perp}(G, M) ; H^{\perp}(G, M)$ is the first cohomology set of $G$ with coefficients in $M$. If $\alpha \in Z^{l}(G, M)$, then $[\alpha] \in H^{l}(G, M)$ denotes the orbit of $\alpha$. Let $1 \in Z^{1}(G, M)$ be the

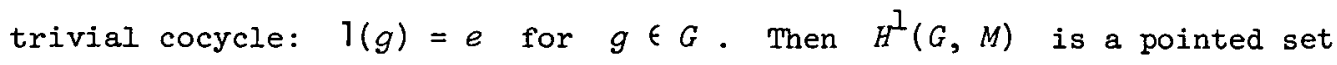
with neutral element [1]. If $M$ is abelian, then $H^{l}(G, M)$ is the usual first cohomology group of the algebraic group $G$ with coefficients in the $G$-group $M$. In this case, we sometimes denote [1] by 0 .

LEMMA 6.2.1. Let $F$ be a finite group whose order is relatively prime to $p$ and let $V$ be an abelion unipotent F-group. Then $H^{l}(E, V)=0$.

Proof. Let $\alpha \in 2^{l}(E, V)$ and let $r=|F|$. Let $\alpha^{r}$ denote the cocycle defined by $\alpha^{r}(f)=\alpha(f)^{r}$ for every $f \in F$. By a standard argument (see [15, p. 5.01]), $\alpha^{r}$ is cohomologous to the trivial cocycle 1 . Thus there exists $v \in V$ such that $\alpha(f)^{r}=v(f \cdot v)^{-1}$ for $f \in F$. Now $V$ is a p-group and hence every element of $V$ has a unique rth root. Let $u \in V$ be such that $u^{r}=v$. Then we have $\left(u(f \cdot u)^{-1}\right)^{r}=v(f \cdot v)^{-1}=\alpha(f)^{r}$ for every $f \in F$, and hence $\alpha(f)=u(f \cdot u)^{-1}$ for every $f \in F$. Thus $[\alpha]=0$.

LEMMA 6.2.2. Let $G$ be an algebraic group, let $M$ be a G-group and let $N$ be a closed normal G-stable subgroup of $M$. Then there exists an exact sequence (of pointed sets)

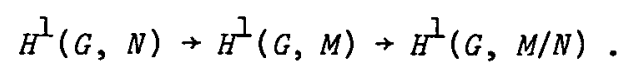

LEMMA 6.2.3. Let $G$ be an algebraic group, let $M$ be a $G$-group. 
and let $H$ be a closed normal subgroup of $G$. Then there exists an exact sequence (of pointed sets)

$$
[1] \rightarrow H^{1}\left(G / H, M^{H}\right) \rightarrow H^{1}(G, M) \rightarrow H^{1}(H, M) \text {. }
$$

For the case of Galois cohomology, results similar to Lemmas 6.2.2 and 6.2.3 are proved in [2; p. 120, Proposition 1.17 and p. 123, Proposition 1.27]. The proofs in our case are essentially the same and will be omitted.

LEMMA 6.2.4. Let $S$ be an algebraic toms and let $V$ be an abelian unipotent $S$-group. Then $H^{\mathcal{I}}(S, V)=0$.

Proof. Let $\alpha \in Z^{l}(S, V)$. Corresponding to the one-cocycle $\alpha$, we define a "twisted" morphic action of $S$ on (the algebraic variety) $V$, denoted by $(s, v) \mapsto s * v$, as follows: $s * v=\alpha(s) s \cdot v$. (See [2, p. 115] for the general setting for this.) We note that $s * v=v$ if and only if $\alpha(s)=v(s \cdot v)^{-1}$. Thus $\alpha$ is cohomologous to the trivial cocycle 1 if and only if the twisted action corresponding to $\alpha$ has a fixed point. Now it is a standard fact that, for any morphic action of an algebraic torus on an affine variety, there are only a finite number of distinct isotropy subgroups. (This is obvious for a linear action on a vector space, and the general case can be reduced to this case.) Let $A_{1}, \ldots, A_{q}$ denote the set of distinct isotropy subgroups for the twisted action of $S$ on $V$. If some $A_{j}=S$, then $S$ has a fixed point on $V$ and hence $\alpha$ is sohomologous to 1 . If not, there exists a finite subgroup $F$ of $S$ such that $F$ is not contained in any $A_{j}$. But $|F|$ is relatively prime to $p$ and consequently, by Lemma 6.2.1, $H^{l}(F, V)=0$; thus $F$ has a fixed point on $V$. This gives a contradiction and proves Lemma 6.2.4.

LEMMA 6.2.5. Let $S$ be a linearly reductive group and $V$ an abelian unipotent $S$-group. Then $H^{1}(S, V)=0$.

Proof. By Lemma 6.2.3, we have an exact sequence

$$
H^{l}\left(S / S^{0}, V^{0}\right) \rightarrow H^{l}(S, V) \rightarrow H^{1}\left(s^{0}, V\right)
$$


But $\left|S / S^{0}\right|$ is relatively prime to $p$ and $S^{0}$ is a torus. Hence, by Lemma 6.2.1, $H^{1}\left(S / S^{0}, V^{0}\right)=0$ and, by Lemma 6.2.4, $H^{1}\left(S^{0}, V\right)=0$. Consequently $H^{1}(S, V)=0$.

LEMMA 6.2.6. Let $S$ be a Zinearly reductive group and $V$ a zonipotent S-group. Then $H^{\mathcal{I}}(S, V)=[1]$.

Proof. By induction on the length $n$ of the derived series for $V$. For $n=1$, the result follows from Lemma 6.2.5. Assume that the result has been proved for the case $n-1$. By Lemma 6.2.2, we have an exact sequence

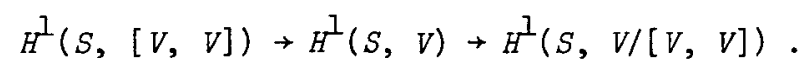

By the inductive hypothesis, $H^{\perp}(S,[v, V])=[1]$ and, by Lemma 6.2.5, $H^{l}(S, V /[V, V])=0$. Thus $H^{1}(S, V)=[1]$.

6.3. We are now ready to prove Proposition 6.1. Let the notation be as in the statement of the proposition. It has been shown by Borel and Tits [4] that $P$ has a Levi subgroup and that any two Levi subgroups of $P$ are conjugate in $P$. Let $L$ be the set of all Levi subgroups of $P$. Then $N_{H}(P)$ acts on $L$ by conjugation. Let $V=R_{u}(P)$ and let $L$ be a Levi subgroup of $P$. Then we claim that $L=N_{P}(L)$. To prove this, it will be sufficient to show that $V \cap N_{P}(L)=\{e\}$. Let $v \in V \cap N_{P}(L)$. Then, for $x \in L$, we have $v x v^{-1} x^{-1} \in L \cap V=\{e\}$. Hence $v \in Z_{V}(L)$. But, if $T$ is a maximal torus of $L$ (hence a maximal torus of $G$ ), it is easy to show that $Z_{V}(T)=\{e\}$ and hence that $Z_{V}(L)=\{e\}$. Thus our claim is proved. Since $P$ acts transitively on $L$ by conjugation and $N_{P}(L)=L$, it follows that $V$ acts simply transitively on $L$.

Now since $S$ normalizes $P, S$ normalizes $V$ and we may consider $V$ as an $S$-group, with $S$ acting by conjugation on $V$. We let $K$ denote the semi-direct product $V \ltimes S$. Now $S$ and $V$ act on (the set) $L$ by conjugation and these actions are compatible; consequently they determine an action of $K$ on $L$ which extends the actions of $S$ and $V$. 
Let $R=\left\{\left.(v, s) \in K\right|^{\left.v s_{L}=L\right\} ; R}\right.$ is the stabilizer of $L$ for the action of $K$ on $L$. Since $V$ acts simply transitively on $L$, we see that the morphism $n: V \rightarrow K / R$ defined by $n(v)=v R$ is a bijection. We wish to show that $n$ is an isomorphism of varieties. It will suffice to show that the differential $d n_{e}$ has trivial kernel. The kernel of $d n_{e}$ is $\underline{\underline{y}} \cap \underline{\underline{r}}$. Let $x \in \underline{\underline{\mathrm{V}}} \cap \underline{\underline{\mathrm{r}}}$. Since $x \in \underline{\underline{\mathrm{v}}}, x$ is a nilpotent element of $\underline{\underline{r}}$. Now it is known that every nilpotent element of $\underline{\underline{r}}$ is tangent to a connected unipotent subgroup of $R^{0}$ [3]. Now $R^{0}$ is contained in the connected solvable group $S^{0} \ltimes V$ and $V$ is the unipotent radical of $S^{0} \ltimes V$. Consequently every unipotent subgroup of $R^{0}$ is contained in $V$. Hence $x$ is tangent to a connected unipotent subgroup of $R^{0} \cap V$. But since $N_{P}(L)=L, R^{0} \cap V=\{e\}$. Therefore $x=0$ and $\eta: V \rightarrow K / R$ is an isomorphism.

Define a morphism $v: S \rightarrow V$ by $v(s)=n^{-1}(s R)$. Thus, for $s \in S$, $v(s)$ is the unique element of $V$ such that $s_{L}=v(s)_{L}$. Moreover, for $s, t \in S$, we have

$$
v(s t)_{L}={ }^{s} t_{L}=s v(t)_{L}=s v(t) s^{-1} s_{L}=(s \cdot v(t)) v(s)_{L} \text {. }
$$

Since $V$ acts simply transitively on $L$, this gives

$$
v(s t)=(s \cdot v(t)) v(s) \text { for } s, t \in S \text {. }
$$

Assume now that there exists $L_{1} \in L$ which is normalized by $S$. We may write $L_{1}=v_{L}$ for some (unique) $v \in V$. Hence, for $s \in S$, we obtain

$$
v_{L}=L_{1}={ }^{s} L_{1}={ }^{s} v_{L}=s v s^{-1} s_{L}=(s \cdot v) \nu(s)_{L}
$$

and consequently, since $V$ acts simply transitively on $L$, we have

$$
v(s)=(s \cdot v)^{-1} v \text { for } s \in S \text {. }
$$

Conversely, if $v$ satisfies $(6.3 .2)$, then $v_{L}=L_{1}$ is normalized by $S$.

Thus we see that, in order to show that $S$ normalizes a Levi subgroup of $P$, it suffices to show that if $v: S \rightarrow V$ is a morphism satisfying 
(6.3.1), then there exists $v \in V$ such that (6.3.2) is satisfied. Let $\nu: S \rightarrow V$ satisfy $(6.3 .1)$ and let $\alpha: S \rightarrow V$ be defined by $\alpha(s)=\nu(s)^{-1}$. Then (6.3.1) implies that $\alpha \in Z^{l}(S, V)$. By Lemma 6.2.6, $H^{l}(s, V)=[1]$. Hence there exists $v \in V$ such that $\alpha(s)=v^{-1}(s \cdot v)$ for $s \in S$. But this implies that $\nu$ satisfies (6.3.2). This proves Proposition 6.1.

\section{A theorem of Kempf}

First we need some notation. If $\varphi: k^{*} \rightarrow X$ is a morphism of algebraic varieties, then we say that $\lim _{t \rightarrow 0} \varphi(t)$ is equal to $x \in X$ if there exists a morphism $\psi: k \rightarrow X$ such that $\psi(t)=\varphi(t)$ for $t \epsilon k^{*}$ and $\psi(0)=x$.

Let $G$ be a (connected) reductive algebraic group, let $T$ be a maximal torus of $G$ and let $W=N_{G}(T) / T$ be the corresponding Weyl group.

A (multiplicative) one-parameter subgroup of $G$ is a homomorphism $\lambda: k^{*} \rightarrow G$ of algebraic groups. We let $X_{*}(G)$ denote the set of oneparameter subgroups of $G$. Since $T$ is abelian, the set $X_{*}(T)$ has a matural structure of abelian group; in fact $X_{*}(T)$ is a free abelian group of rank equal to the dimension of $T$. The group $G$ acts on the set $X_{*}(G)$ via inner automorphisms: if $\lambda \in X_{*}(G)$ and $g \in G$, then the oneparameter group $g \cdot \lambda$ is defined by $(g \cdot \lambda)(t)=g \lambda(t) g^{-1}$. Similarly $W$ acts on $X_{*}(T)$.

If $\lambda \in X_{*}(G)$, let $P(\lambda)=\left\{g \in G \mid \lim _{t \rightarrow 0} \lambda(t) g \lambda(t)^{-1}\right.$ exists $\}$. Then $P(\lambda)$ is a parabolic subgroup of $G\left[10, \mathrm{p}\right.$. 55]. If $\lambda \in X_{*}(T)$ and if $\Phi=\Phi(T, G)$ is the set of roots of $G$ with respect to $T$, then $P(\lambda)$ is the parabolic subgroup of $G$ containing $T$ which corresponds to the set of roots $\{\alpha \in \Phi \mid\langle\alpha, \lambda\rangle \geq 0\}$ (see [19, p. 320, Proposition 3]). If $\lambda \in X_{*}(G)$ and $M$ is a closed subgroup of some algebraic group containing $G$, we often write $Z_{M}(\lambda)$ for $Z_{M}$ (image $\lambda$ ). It is well-known [4] that $Z_{G}(\lambda)=Z_{P(\lambda)}(\lambda)$ and that $Z_{P(\lambda)}(\lambda)$ is a Levi subgroup of $P(\lambda)$.

Now let $G$ act morphically on an affine algebraic variety $X$ and let $x \in X$. It is a consequence of the Mumford Conjecture that there is a 
unique closed orbit $C$ in the closure of the orbit $G \cdot x$ (see [14, Lemma 1.4]). It follows from (a minor extension of) the Hilbert-Mumford Theorem (see [8, Theorem 1.4]) that there exists $\lambda \in X_{*}(G)$ such that $\lim \lambda(t) \cdot x$ exists and is a point of $C$. Let $\Lambda(x)$ denote the set of all $\lambda \in X_{*}(G)$ such that $\lim _{t \rightarrow 0} \lambda(t) \cdot x$ exists and is a point of $C$. Kemp $[8]$ has shown that there exists a (non-empty) "optimal" class $\mathcal{C}(x) \subset \Lambda(x)$ which has the following properties:

(7.1). (i) If $\lambda, \mu \in \mathcal{C}(x)$, then $P(\lambda)=P(\mu)$.

(ii) Let $P=P(x)$ denote $P(\lambda)$ for some (and hence every) $\lambda \in \mathcal{C}(x)$. Then $C(x)$ is a complete $P$-orbit in $X_{*}(P)$, that is $\mathcal{C}(x)=P \cdot \lambda$ for any $\lambda \in \mathcal{C}(x)$.

(iii) If $g \in G$, then $\mathcal{C}(g \cdot x)=g \cdot \mathcal{C}(x)$ and hence $g_{P(x)}=P(g \cdot x)$. In particular $G_{x} \subset N_{G}(P)=P$.

Roughly speaking, the optimal class consists of those $\lambda \in \Lambda(x)$ for which $\lambda(t) \cdot x$ approaches $C$ most rapidly as $t$ approaches 0 . The optimal class $\mathcal{C}(x)$ depends on the choice of a $W$-invariant, integervalued, positive-definite, symmetric bilinear form on $X_{*}(T)$. Such a form uniquely determines a $G$-invariant "norm" on $X_{*}(G)$; see [8] for definition and details. Once such a $W$-invariant form on $X_{*}(T)$ is chosen, the optimal class $\mathcal{C}(x)$ is uniquely determined for every $x \in X$ by the geometric data.

We wish to extend 7.1 (iii) above to a slightly more general setting. Let $H$ be an algebraic group and $G$ a closed, connected, normal subgroup of $H$ such that $G$ is a reductive group. Let $T$ be a maximal torus of $G$, let $W=N_{G}(T) / T$ be the corresponding Weyl group and let $W_{1}=N_{H}(T) / Z_{H}(T)$. By the rigidity of tori, $W_{1}$ is a finite group. Moreover, $W$ is canonically embedded as a normal subgroup of $W_{1}$. The group $H$ acts on $X_{*}(G)$ by conjugation and $W_{1}$ acts similarly on $X_{*}(T)$. We let $X_{*}(G) / H$ (respectively $X_{*}(T) / W_{1}$ ) denote the set of orbits of $H$ on $X_{*}(G)$ (respectively of $W_{1}$ on $X_{*}(T)$ ).

LEMMA 7.2. The inclusion map $X_{*}(T) \rightarrow X_{*}(G)$ induces a bijection $B$ 
of $\left.X_{*}(T) / W_{1} \rightarrow X_{*} ! G\right) / H$

Proof. Let $\lambda \in X_{*}(G)$. Then image $(\lambda)$ is $G$-conjugate to a subtorus of $T$. Thus there exists $g \in G$ such that $g \cdot \lambda \in X_{*}(T)$. Hence $\beta$ is surjective. Now let $\mu, \nu \in X_{*}(T)$ and assume that there exists $h \in H$ such that $h \cdot \mu=\nu$. Then $T$ and $h_{T}$ both contain image $(\nu)$, hence $T$ and $h_{T}$ are both maximal tori of $z_{G}(v)$. Therefore, there exists $g \in Z_{G}(\nu)$ such that $g h_{T}=T$. Thus $g h \in N_{H}(T)$. We let $W$ denote the image of $g h$ in $W_{1}$. Then $w \cdot \mu=(g h) \cdot \mu=g \cdot \nu=\nu$. This shows that $B$ is injective.

Since $W_{1}$ is finite, we may choose a $W_{1}$-invariant, integer-valued, positive definite, symmetric bilinear form on $X_{*}(T)$. As in Kempf [8], this extends to a $G$-invariant norm on $X_{*}(G)$. It follows trivially from Lemma 7.2 that this norm on $X_{*}(G)$ is $H$-invariant.

Now let $H$ act morphically on the affine variety $X$ and let $C$ denote the unique closed orbit in the closure of $G \cdot x$. Considering $X$ as a $G$-variety, we may define $\Lambda(x), \mathcal{C}(x)$ and $P(x)$ as above; $\mathcal{C}(x)$ and $P(x)$ are defined with respect to an $H$-invariant norm on $X_{*}(G)$ constructed as above.

LEMMA 7.3. Let the notation be as above. Then the optimal class $\mathcal{C}(x)$ is stable under the action of the isotropy subgroup $H_{x}$. In particular, $H_{x}$ normalizes $P(x)$.

Proof. This follows immediately from Kempf's definition of the optimal class $C(x)$ and the fact that the norm on $X_{*}(G)$ is $H$-invariant.

\section{Proof of Theorem $\mathrm{C}$}

Let the notation be as in the statement of Theorem $C$. We choose an $H$-invariant norm on $X_{*}(G)$ as indicated in section 7 . Let $C(x)$ be the optimal class as given by Kempf's theorem and let $P=P(x)$ be the corresponding parabolic subgroup. Since $x \in x^{S}$, it follows from Lemma 7.3 that $\mathcal{C}(x)$ is stable under the action of $S$ and that $S$ normalizes $P$. Let $\lambda \in \mathcal{C}(x)$. Then $D(\lambda)=P$ and $L=Z_{G}(\lambda)$ is a Levi subgroup of 
$P$. Let $L$ denote the set of all Levi subgroups of $P$. We define a map $F: C(x) \rightarrow L$ by $F(\mu)=Z_{G}(\mu)$ for $\mu \in C(x)$.

LEMMA 8.1. $F$ is a bijection.

Proof. If $g \in P$ and $\mu \in \mathcal{C}(x)$, then

$$
F(g \cdot \mu)=Z_{G}(g \cdot \mu)=g_{G}(\mu)=g_{F(\mu)} .
$$

Thus $F$ is a $P$-mapping of $P$-sets. Now $P$ acts transitively on $\mathcal{C}(x)$ and on $L$ and hence $F$ is a surjection. Assume that $g \in P$ is such that

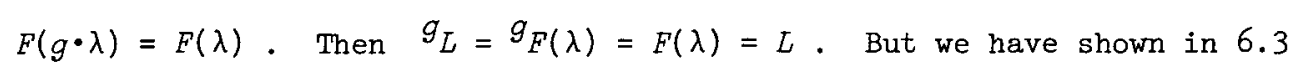
that $N_{P}(L)=L$. Hence $g \in L=Z_{P}(\lambda)$. Thus $\lambda$ and $F(\lambda)$ have the same isotropy subgroups for the actions of $P$. Since $F$ is a $P$-mapping of transitive $P$-sets, this implies that $F$ is a bijection.

Since $S$ normalizes $P$, we see by Proposition 6.1 that there exists a Levi subgroup $L_{1}$ of $P$ which is normalized by $S$. We may write $L_{1}=F(\mu)=Z_{G}(\mu)$ for some $\mu \in \mathcal{C}(x)$. Let $s \in S$. Then, by Lemma 7.3 , $s \cdot \mu \in \mathcal{C}(x)$ and we have

$$
F(s \cdot \mu)=Z_{G}(s \cdot \mu)={ }^{s} Z_{G}(\mu)={ }^{s} L_{1}=L_{1}=Z_{G}(\mu)=F(\mu) .
$$

Since $F$ is a bijection, this implies that $s \cdot \mu=\mu$. Hence we see that $s \mu(t) s^{-1}=\mu(t)$ for every $t \in k^{*}$. Consequently $\mu \in X_{*}\left(\left(G^{S}\right)^{0}\right)$. This gives the first conclusion of Theorem $C$. In particular, we see that if $G \cdot x$ is not closed, then $Z \cdot x$ is not closed. For the converse, assume that $G \cdot x=X_{1}$ is closed. Then $H_{1}=G S$ is a homomorphic image of the semi-direct product $G \ltimes S$ and hence is a closed subgroup of $H$. Since $x \in X^{S}$, we see that $H_{1} \cdot x=G \cdot x=X_{1}$. It follows from Theorem $A$, as applied to the action of $H_{1}$ on $X_{1}$, that $2 \cdot x$ is closed in $X_{1}$. Since $X_{1}$ is closed in $X$, we see that $2 \cdot x$ is closed in $X$. This completes the proof of Theorem $C$.

\section{Applications to involutions of algebraic groups}

In this section, we assume that characteristic $(k) \neq 2$. 
In this section we will prove several results concerning orbits which are associated to involutions of algebraic groups. There results will be used in a forthcoming paper of the author on involutions of reductive groups.

Let $G$ be a connected algebraic group and let $\theta$ be an automorphism of $G$ of period two. We consider two morphic actions of $G$ on (the algebraic variety) $G$ :

(1) The action of $G$ by conjugation: $g \cdot x=g x g^{-1}$ for $g, x \in G$. Here the orbit $G \cdot x$ is just the conjugacy class $C_{G}(x)$.

(2) The "twisted" action of $G$ on $G$, denoted by $(g, x) \mapsto g \star x$, where $g * x=g x \theta(g)^{-1}$. We denote the G-orbit of $x$ for the twisted action of $G$ by $G * x$.

Let $K=G^{\theta}$. We note that, for $k \in K$ and $x \in G$, we have $k \cdot x=k * x$. We let $P=G * e=\left\{g \theta(g)^{-1} \mid g \in G\right\}$ and let $Q=\left\{g \in G \mid \theta(g)=g^{-1}\right\}$. Then $Q$ is a closed subvariety of $G$ which is stable under the twisted action of $G$ and $P$ is contained in $Q$. Also $Q$ is $\theta$-stable.

We will prove the following propositions:

PROPOSITION 9.1. Let $G$ act on $Q$ via the twisted action. Then $G$ acts transitively on each connected component of $Q$. Hence $Q$ is $a$ finite union of twisted G-orbits and each twisted G-orbit on $Q$ is closed in $G$. In particular, $P$ is closed in $G$.

PROPOSITION 9.2. Let $y \in Q$. Then $K^{O}$ acts transitively on each connected component of $Q \cap C_{G}(y)$. In particular, $Q \cap C_{G}(y)$ is a finite union of $K^{0}$-orbits, each of which is closed in $Q \cap C_{G}(y)$.

PROPOSITION 9.3. Let $G$ be reductive.

(a) The following conditions on $y \in P$ are equivalent:

(i) $y$ is semisimple;

(ii) $C_{G}(y)$ is closed in $G$; and 
(iii) $K^{0} \cdot y=C_{K}(y)$ is closed in $P$.

(b) The following conditions on $y \in P$ are equivalent:

(iv) $y$ is unipotent;

(v) $e$ belongs to the closure of $C_{G}(y)$; and

(vi) $e$ belongs to the closure of $k^{0} \cdot y$.

Proof of Proposition 9.1. We define an action of $G \times G$ on (the algebraic variety) $G$ by $\left(g_{1}, g_{2}\right) \cdot x=g_{1} x \theta\left(g_{2}\right)^{-1}$. Let $\Delta(G)$ denote the diagonal subgroup of $G \times G: \Delta(G)=\{(g, g) \mid g \in G\}$. Then the $\Delta(G)-$ orbit of $x \in G$ is just the twisted orbit $G * x$. Define an involutive automorphism $s$ of $G \times G$ by $s\left(g_{1}, g_{2}\right)=\left(g_{2}, g_{1}\right)$. Let $s$ denote the two-element subgroup of $\operatorname{Aut}(G \times G)$ consisting of the identity automorphism of $G \times G$ and $s$. Let $H$ denote the semi-direct product of $G \times G$ and $S$. We identify $G \times G$ and $S$ with closed subgroups of $H$ in the usual way. Since characteristic $(k) \neq 2, S$ is a linearly reductive group. Clearly $\left(H^{S}\right)^{0}=\Delta(G)$. We define an action of $S$ on the algebraic variety $G$ by $s \cdot x=\theta(x)^{-1}$ for $x \in G$. Then, for $g_{1}, g_{2}, x \in G$, we have

$$
\begin{aligned}
s \cdot\left(\left(g_{1}, g_{2}\right) \cdot x\right) & =s \cdot\left(g_{1} x \theta\left(g_{2}\right)^{-1}\right) \\
& =g_{2} \theta(x)^{-1} \theta\left(g_{1}\right)^{-1} \\
& =\left(g_{2}, g_{1}\right) \cdot(s \cdot x) \\
& =\left(s \cdot\left(g_{1}, g_{2}\right)\right) \cdot(s \cdot x) .
\end{aligned}
$$

Thus the actions of $G \times G$ and $S$ on $G$ are compatible and determine an action of the semi-direct product $H$ on $G$. We note that $G$ (the set of fixed points of $S$ on $G$ by the action defined above) is equal to $Q$. Hence, by Theorem A (with the $X$ of Theorem A equal to $G$ above and with $\dot{G}$ and $H$ of Theorem A equal to $H^{0}$ and $H$ above), we see that $\Delta(G)$ acts transitively on each connected component of $Q$. Since $\Delta(G) \cdot x=G * x$ for $x \in G$, the proposition is proved. 
Proof of Proposition 9.2. For the proof of this proposition, we let $S$ denote the two-element subgroup $\left\{1_{G}, \theta\right\}$ of $\operatorname{Aut}(G)$ and let $H$ denote the semi-direct product of $G$ and $S$. Let $G$ act on $G$ by conjugation. Thus $G \cdot x=C_{G}(x)$ for $x \in G$. We define an action of $S$ on the algebraic variety $G$ by $\theta \cdot x=\theta(x)^{-1}$ for $x \in G$. With this action of $S$ on $G$, the set of fixed points of $S$ on $G$ is exactly $Q$. We have

$$
\begin{aligned}
\theta \cdot(g \cdot x) & =\theta \cdot\left(g x g^{-1}\right) \\
& =\theta(g) \theta(x)^{-1} \theta(g)^{-1} \\
& =\theta(g) \cdot(\theta \cdot x) .
\end{aligned}
$$

Thus the actions of $G$ and $S$ on $G$ are compatible and determine an action of the semi-direct product $H$ on $G$. Let $y \in Q$ and let $X=G \cdot y=C_{G}(y)$. Then $\theta \cdot(G \cdot y)=G \cdot(\theta \cdot y)=G \cdot y$ and hence $X$ is

H-stable. How $K^{0}=\left(G^{S}\right)^{0}$ and $X^{S}=C_{G}(y) \cap Q$. Since characteristic $(k) \neq 2$, the action of $S$ on $\underline{\underline{g}}$ is semisimple. The conclusion of Proposition 9.2 now follows from Theorem $A$.

Proof of Proposition 9.3. Let $H$ and the action of $H$ on $G$ be defined as in the proof of Proposition 9.2. To conform with the notation of Theorem C, let $G=X$ (as the algebraic variety on which $H=G \ltimes S$ acts). Then $X^{S}=Q$ and $K^{0}=\left(G^{S}\right)^{0}$. It follows from theorem $c$ that $K^{0} \cdot y$ is closed if and only if $G \cdot y=C_{G}(y)$ is closed. This proves the equivalence of $(i i)$ and $(i i i)$. Theorem $C$ also implies the equivalence of (v) and (vi). The equivalence of (i) and ( $i i)$ and the equivalence of ( $i v$ ) and $(v)$ are well known [17]. This proves Proposition 9.3.

9.4. Now let $G$ be a connected Lie group and let $\theta$ be an involutive automorphism of $G$. Let $P, Q$ and the twisted action of $G$ on $G$ be defined as above. Then we have:

PROPOSITION 9.4.1. The twisted action of $G$ is transitive on each connected component of $Q$. In particular, $Q$ is a closed differentiable submanifold of $G$ and each twisted $G$-orbit on $Q$ is closed. Hence $P$ is closed.

The proof is a precise Lie group analogue (using Theorem B) of 
Proposition 9.1. We omit the details. One can, in fact, show that $Q$ has only a finite number of connected components, but we shall not give the proof here.

\section{Some more applications}

10.1. Affine orbits and reductive subgroups. We will use the following characterization of reductive subgroups of reductive groups:

10.1.1. Let $H$ be a closed subgroup of the reductive algebraic group $G$. Then $H^{0}$ is a reductive group if and only if the coset space $G / H$ is an affine variety.

See [14] for a proof.

Before giving our applications, we need a few elementary lemmas.

LEMMA 10.1.2. Let $S$ be a subgroup of GL(E) such that $E$ is a semisimple s-module. Then $\mathrm{GL}(E)^{S}$ is a reductive group.

Proof. Let $A$ denote the commuting algebra of $S$ in $\operatorname{End}_{k}(E)$. Then $A$ is a direct product of matrix algebras. (This is a standard result in the theory of semisimple associative algebras. See, for example, $\left[7\right.$, p. 79].) Hence $\mathrm{GL}(E)^{S}$ is isomorphic to a product of the form $\mathrm{GL}_{m_{\perp}}(k) \times \ldots \times \mathrm{GL}_{m_{r}}(k)$. In particular $\mathrm{GL}(E)^{S}$ is reductive.

LEMMA 10.1.3. Let the algebraic group $G$ act morphically on the affine algebraic variety $X$ and let $x \in X$ be such that the orbit $G \cdot x$ is closed. Then $G / G_{x}$ is an affine variety.

Proof. The orbit map $\varphi_{x}: G \rightarrow G \cdot x$ determines a bijective morphism $\psi: G / G_{x} \rightarrow G \cdot x$. Since $G / G_{x}$ and $G \cdot x$ are normal varieties, it follows from Zariski's Main Theorem that $\psi$ is a finite morphism (see [5, p. 138, Corollary 3]). Since $G \cdot x$ is an affine variety, this implies that $G / G_{x}$ is an affine variety.

LEMMA 10.1.4. Let $H$ and $K$ be closed subgroups of an algebraic group $G$, let $\pi_{H}: G \rightarrow G / H$ and $\pi_{K}: G \rightarrow G / K$ be the canonical morphisms 
and let $a=\pi_{H}(e), b=\pi_{K}(e)$. Let $G$ aet on $G / H$ and $G / K$ by left translations. If the orbit $K \cdot a$ is closed in $G / H$, then $H \cdot b$ is closed in $G / K$.

Proof. If $K \cdot a$ is closed in $G / H$, then $K H=\pi_{H}^{-1}(K \cdot a)$ is closed in $G$ and therefore $H K=(K H)^{-1}$ is closed in $G$. But the topology on $G / K$ is the quotient topology. Consequently, since $H K=\pi_{K}^{-1}(H \cdot b)$ is closed in $G$, we see that $H \cdot b$ is closed in $G / K$.

If $S$ is a reductive subgroup of a reductive algebraic group $G$ and if $k$ is of characteristic zero, then it is known that ${ }_{G}(S)^{0}$ and $N_{G}(S)^{0}$ are reductive groups. This result is not true in prime characteristic $p$. However, the following result shows that it does hold if $S$ is linearly reductive.

PROPOSITION 10.1.5. Let $S$ be a linearly reductive group and let $G$ be a connected $S$-group. Then $G / G^{S}$ is an affine variety. In particular, if $G$ is reductive, then $\left(G^{S}\right)^{0}$ is reductive. If $S$ is a ciosed linearly reductive subgroup of $G$ acting on $G$ by inner automorphisms, then $N_{G}(S)^{0}=\left(S G^{S}\right)^{0}$. If, in addition, $G$ is reductive, then $N_{G}(S)^{0}$ is reductive.

Proof. Let $H$ denote the semi-direct product $G \ltimes S$. We consider $G$ and $S$ as closed subgroups of $H$ in the usual way. We may embed $H$ as a closed subgroup of some $\mathrm{GL}(E)$. By Lemma 10.1.2 we see that $M=\mathrm{GL}(E)^{S}$ is a reductive group. Since $S$ normalizes $G$, it follows from Section 2 that we may define a morphic action of $S$ on $\operatorname{GL}(E) / G=X$ by $s \cdot(g G)=s g s^{-1} G$. (Warning: this is not necessarily the same as the action of $S$ on $G L(E) / G$ by left translation.) We consider $G L(E)$ as an $S$-group, with $S$ acting by inner automorphisms. Let GL(E) act on $X$ by left translations. Then the actions of $G L(E)$ and $S$ on $X$ are compatible and hence define an action of the semi-direct product GL(E) $\propto S$ on $X$. Let $\pi: G L(E) \rightarrow G L(E) / G=X$ be the canonical map and let $a=\pi(e)$. To avoid confusing notation, let $S^{\prime}$ denote the subgroup 
$\{e\} \times S$ of $G L(E) \propto S$. We now apply Theorem $A$ to the above situation, with the $H$ (respectively $G, S$ ) of Theorem A replaced by GL $(E) \propto S$ (respectively $G L(E), S^{\prime}$ ) above. By Theorem $A$, every orbit of $M$ on $X^{\prime}$ is closed. In particular $M \cdot a$ is closed. Let $\pi^{\prime}: \mathrm{GL}(E)+\mathrm{GL}(E) / M$ be the canonical map and let $b=\pi^{\prime}(e)$. Since $M \cdot a$ is closed, we see from Lemma 10.1 .4 that $G \cdot b$ is closed for the action of $G$ on $G L(E) / M$ by left translations. Since $M$ is reductive, it follows from 10.1 .1 that $\mathrm{GL}(E) / M$ is an affine variety. Thus, by Lemma 10.1 .3 , we see that $G / G_{b}$ is an affine variety. But $G_{b}=G \cap M=G^{S}$ and consequently $G / G^{S}$ is an affine variety. If $G$ is reductive, then 10.1 .1 implies that $\left(G^{S}\right)^{0}$ is reductive.

Assume now that the linearly reductive group $S$ is a closed subgroup of $G$ acting on $G$ by inner automorphisms. We let $G$ act on $X_{1}=G / S$ by left translation and let $S$ act on $X_{1}$ as a subgroup of $G$. Let $\pi_{1}: G \rightarrow G / S=X_{1}$ be the canonical map and let $c=\pi_{1}(e)$. Then $c \in X_{1}^{S}$. By Theorem A, $Z=\left(G^{S}\right)^{0}$ acts transitively on the connected component $Y$ of $X_{1}^{S}$ which contains $c$. Let $N=N_{G}(S)^{0}$. Clearly $Y$ is $N$-stable. Hence $N \cdot C$ is contained in $Z \cdot C=Y$. Thus we see that, for $n \in N$, we have $n S=z S$ for some $z \in Z$ and hence $n \in Z S$. Thus we have shown that $N=(Z S)^{0}$. Assume further that $G$ is reductive. By the proof above, $Z$ is reductive. Moreover, $N / Z=\left(Z S^{0}\right) / Z$ is a homomorphic image of the reductive group $S^{0}$ and hence is a reductive group. Since $Z$ and $N / Z$ are reductive groups, it follows easily that $N$ is a reductive group. This proves Proposition 10.1.5.

COROLLARY 10.1.6. Let $S$ be a linearly reductive group, let $G$ be a connected S-group and let $Z=(G)^{0}$. Let $L$ be a closed s-stable subgroup of $G$ such that the coset space $G / L$ is an affine variety. Then $Z / Z \cap L$ is an affine variety.

Proof. Let $\pi: G \rightarrow G / L$ be the canonical map and let $a=\pi(e)$. By 
Theorem A, the orbit $Z \cdot a$ is closed in the affine variety $G / L$. Consequently, by Lemma 10.1.3, $2 / Z_{a}$ is an affine variety and clearly $Z_{a}=Z \cap L$

10.2. Complete orbits and parabolic subgroups.

PROPOSITION 10.2.1. Let $S$ be a linearly reductive group, let $G$ be a connected $S$-group and let $Z=(G)^{0}$. Let $P$ be an s-stable parabolic subgroup of $G$ and let $X=G / P$. Let $G$ act on $X$ by left translation and let $S$ act on $X$ by $s^{\cdot}(g P)=\left(s^{*} g\right) P$. Then $Z$ acts transitively on each irreducible component of $X^{S}$. In particular, $Z$ has only a finite number of orbits on $x^{S}$ and each such orbit is a complete variety. Let $P$ denote the set of subgroups of $G$ which are $G$-conjugate to $P$. Then $P$ is stable under the natural action of $S$ on subgroups of $G$. If $Q \in P^{S}$, then $Z \cap Q$ is a parabolic subgroup of $Z$.

Proof. Since $S$ and $G$ act compatibly on $X$, these actions determine a morphic action of the semi-direct product $H=G \propto S$ on $X$ as in Section 2. Consequently, by Theorem A, $Z$ acts transitively on each irreducible component of $X$. The other conclusions of Proposition 10.2.1 now follow easily.

REMARKS. (i) For the case of Proposition 10.2 .1 in which $S$ is a torus subgroup of $G$ acting by inner automorphisms and $P$ is Borel subgroup of $G$ containing $S$, we recover a result of Chevalley (see [1, p. 279, 11.18]).

(ii) The example of 3.3 shows that, in Proposition 10.2.1, it is not necessarily the case that all orbits of $z$ on $x^{S}$ have the same dimension.

10.3. Compact orbits and uniform subgroups. We recall that a closed subgroup $L$ of the Lie group $G$ is uniform if the coset space $G / L$ is compact.

PROPOSITION 10.3.1. Let $G$ be a connected Lie group and let $S$ be a subgroup of $\operatorname{Aut}(G)$ such that the representation of $S$ on $\mathrm{g}$ is semisimple. Let $L$ be an S-stable uniform subgroup of $G$, let $G$ act 
on $X=G / L$ by left translations and let $S$ act on $X$ by $s \cdot(g L)=(s \cdot g) L$. Then $X^{S}$ is a closed differentiable submonifold of $X$ and $z=\left(G^{S}\right)^{0}$ acts transitively on each connected component of $x^{S}$. In particular, $Z$ has only a finite number of orbits on $x^{S}$ and each such orbit is compact. Moreover $Z \cap L$ is a uniform subgroup of $Z$.

Proof. Let $N=\{a \in \operatorname{Aut}(G) \mid a \cdot L=L\}$. We let $N$ act on $X$ by $a \cdot(g L)=(a \cdot g) L$. Then the actions of $N$ and $G$ on $X$ are compatible and hence determine an action of $H=G \times N$ on $X$. The proof of Proposition 10.3.1 now follows immediately from Theorem B.

\section{References}

[1] Armand Borel, Linear algebraic groups (Benjamin, New York, Amsterdam, 1969).

[2] A. Borel et J.-P. Serre, "Théorèmes de finitude en cohomologie galoisienne", Comment. Math. HeZv. 39 (1964/1965), 111-164.

[3] A. Borel and T.A. Springer, "Rationality properties of linear algebraic groups II", Tôhoku Math. J. 20 (1968), 443-497.

[4] Armand Borel et Jacques Tits, "Groupes rédictifs", Inst. Hautes Êtudes Sci. Publ. Math. 27 (1965), 55-150.

[5] J. Dieudonné, Cours de géométrie algebrique, 2 (Presses universitaires de France, Paris, 1974).

[6] J. Dieudonné, Éléments d'analyse. Tome III: Chapitres XVI et XVII (Cahiers Scientifiques, Fasc. 33. Gauthier-Villars Éditeur, Paris, 1970).

[7] Jean A. Dieudonné and James B. Carrell, "Invariant theory, old and new", Adv. in Math. 4 (1970), 1-80.

[8] George R. Kempf, "Instability in invariant theory", Ann. of Math. (2) 108 (1978), 299-316.

[9] D. Luna, "Adhérences d'orbite et invariants", Invent. Math. 29 (1975), 231-238. 
[10] David Mumford, Geometric invariant theory (Ergebnisse der Mathematik und ihrer Grenzgebiete, 34. Springer-Verlag, Berlin, Heidelberg, New York, 1965).

[11] Masayoshi Nagata, "Complete reducibility of rational representations of a matric group", J. Math. Kyoto Univ. 1 (1961-62), 87-99.

[12] M.S. Raghunathan, Discrete subgroups of Lie groups (Ergebnisse der Mathematik und ihrer Grenzgebiete, 68. Springer-Verlag, Berlin, Heidelberg, New York, 1972).

[13] R.W. Richardson, "Conjugacy classes in Lie algebras and algebraic groups", Ann. of Math. (2) 86 (1967), 1-15.

[14] R.W. Richardson, "Affine coset spaces of reductive algebraic groups", Buzz. London Math. Soc. 9 (1977), 38-41.

[15] J-P. Serre, "Applications algébriques de la cohomologie des groupes. I", Cohomologie des groupes, suite spectrale, faisceaux, Exposé 5 (Séminaire Henri Cartan de L'Ecole Normale Supérieure, 3e année, 1950/1951. Secrétariat Mathématique, Paris, 1955).

[16] T.A. Springer, Linear algebraic groups (Birkhauser, Boston, Basel, Stuttgard, 1981).

[17] Robert Steinberg, "Regular elements of semisimple algebraic groups", Inst. Hautes Etudes Sci. Publ. Math. 25 (1965), 49-80.

[18] Э.Е. Винберг [Ë.В. Vinberg], "Группа Вейля градунрованной алгебры Лн" [The Weyl group of a graded Lie algebra], Izv. Akad. Nauk SSSR 40 (1976), 488-526. English translation: Math. USSR-Izv. $10(1976), 463-495$ (1977).

[19] Thierry Vust, "Opération de groupes réductifs dans un type de cônes presque homogènes", Bulz. Soc. Math. France 102 (1974), 317-333.

[20] Hassler Whitney, "Elementary structure of real algebraic varieties", Ann. of Math. (2) 66 (1957), 545-556.

Department of Mathematics, Institute of Advanced Studies, Australian National University, PO Box 4, Canberra, ACT 2600, Australia. 